\title{
Molecular epidemiology of Bombyx mori nucleopolyhedrovirus in Yunnan sericulture region, China
}

\author{
Yonghong Zhang, Fenfen Tang, Yulan Shao, Feng Zhu, Xingrong Bai* \\ Institute of Sericulture and Apiculture, Yunnan Academy of Agricultural Science, Mengzi Yunnan 661101, \\ China \\ *Corresponding author, e-mail: bxrong3@163.com
}

Received 7 Dec 2017

Accepted 31 Aug 2019

\begin{abstract}
Sericultural production is seriously influenced by the blood pathogens of grasserie, Bombyx mori nucleopolyhedrovirus (BmNPV). To investigate the infectious condition and define an effective control method for BmNPV. 233 from 240 suspected samples infected with BmNPV were detected using PCR identification. One sample was selected from each county in the positive sample collection area, 56 isolates of which were used as experimental objects. The homology of the bro- $d$ nucleotide sequence was at least $91 \%$ among the 56 BmNPV isolates. However, the conserved 7 amino acids (percentage of amino acid constant sites, PAACS) at the position 217-224 were absent for YNQJLL001 and YNZTQJ074 pandemic isolates. Phylogenetic tree analysis of bro-d gene separated the BmNPV isolates from Yunnan into 4 main clades: I, II, III, and IV. Clade I was the dominant clade containing 32 local epidemic isolates. Clades II, III, and IV contained 5, 16, and 3 pandemic isolates, respectively. Geographical distribution of clade I in Yunnan showed a concentrated pattern, while the remaining members of the other three clades depicted no direct contact in relation to their locations. This study may provide valuable molecular types on BmNPV isolate distribution in the silkworm-raising regions, also be helpful for evaluating BmNPV pathogenesis and epidemic situations in the Yunnan sericulture region.
\end{abstract}

KEYWORDS: Bombyx mori nucleopolyhedrovirus, bro-d, evolutionary analysis, BmNPV

\section{INTRODUCTION}

Bombyx mori nucleopolyhedrovirus (BmNPV) belongs to the baculovirus division (Baculoviridae), with inclusions in the true baculovirus subfamily (Eubaculovirinae) karyotype polygonal body virus (nucleopolyhedrovirus) ${ }^{1}$. Silkworm is infected by BmNPV, causing blood grasserie, and its first symptom is a colour change of the epidermis from white to yellowish white, followed by the swelling of body segments, and finally to a state of purulence and death ${ }^{2}$. The disease causes serious harm in sericulture-prevalent regions of the world. While this disease occurs in varying seasons among some sericultural regions of China, it particularly affects the Yunnan region during the summer. This accounts for $70-90 \%$ of the total loss in cocoon production $^{3}$. The average incidence rate is about $5 \%$ in Yunnan sericulture regions. The disease incidence has risen in recent years; for example, some regions have experienced an incidence rate of more than $13 \%{ }^{4}$.

BmNPV can generate two kinds of virus particles in the developmental cycle. A sprouting circulation type virus (budded virions, BV) and an embedding virus (occluded virions, ODV) alternately infect the silkworm completely ${ }^{5}$. The silkworm lacks immunity required to combat this two-phase attack of alternating infection. Hence the study of BmNPV itself is focused on its prevention and cure. In recent years, the research of BmNPV genome and functional gene identification showed significant progress. However, few reports exist that focus on transgenerational BmNPV genetic traits from isolated geographical environments (e.g., morphology, pathogenicity, serological reaction, biological physicochemical properties, and so on) and on determining whether differences between BmNPV from various ecological and geographic regions exist, or if they are identical strains. A study performed by Qi found that the $v p 39$ gene homology of BmNPV isolates from China and Japan were above $98 \%{ }^{6}$, and polyhedra production and gene structures showed differences among the three BmNPV isolates were from Korean farm ${ }^{7}$. The evolution 
analysis found that Guangxi BmNPV isolates were divided into three groups based on the polyhedrin and $p 10$ genes, which suggested that the Guangxi sericulture regions had distributed with different pandemic strains ${ }^{8}$.

Some DNA viruses are diverse due to their high levels of adaptability and rapid evolution ${ }^{9}$. Baculoviruses pathogenic to lepidopteran larvae (silkworms) exhibit considerable genetic diversity. BmNPV run parallel with one another in genome evolution ${ }^{10}$ and host selection pressure results in either a BmNPV gene loss or increase. Some genes of BmNPV are deleted or increased, thus improving the ability of virus to infect the host and adapt to the environment and result in viral genome diversity. Genome evolution is mainly caused by gene duplication and horizontal gene transfer ${ }^{11}$. Gene duplication creates BmNPV diversity between genome strains ${ }^{12}$. The bro (baculovirus repeated open reading frames) gene family is typical of the insect baculovirus genome, and they are widely distributed in the insect baculovirus genome. However, gene fragment size and copy number for the type of virus is different. Different bro genes in the virus genome copy numbers were $1-16^{13-16}$, and this is often used to distinguish between BmNPV strains ${ }^{17}$. The bro gene exists in insect baculovirus, which is not only found in the insect iridescent virus (Iridovirus), but with high homologous genes as well ${ }^{18}$. With NPV as the host of the silkworm genome, the bro gene copy number is also different (such as Zhou by Japanese seedlings BmNPV, BmNPV-T3 and BmNPV-Thailand, Chongqing plant BmNPV-CQ1, BmNPV-GD, French seedlings BmNPV-SC7) ${ }^{19}$. The comparative analysis of the different BmNPV strains found that the bro- $a$, bro-b, bro-c, bro-d, bro-e 5 gene copies were present in the BmNPV-T3 strain, where the bro- $a$ gene was present in one region of the genome alone, and bro$b$ and $b r o-c$ were present in a region of the genome in series. Bro-d and bro-e are also present in the other region of the genome in series. Bro genes are highly conserved in many BmNPV genomes. The percentage of amino acid conserved sites (PAACS) of bro gene in the genome of different BmNPV strains was over $82 \%$. The bro- $a$, bro-c, and bro- $d$ genes make up $83 \%, 87 \%$, and $83 \%$, respectively ${ }^{20}$. Deletion mutants of the bro gene constructed in the BmNPV genome in which the bro- $a, b r o-b, b r o-c$, and bro-e genes individually inactivated are successfully isolated. However, exhaustive efforts failed to isolate a bro-d deficient mutant. It may be possible to isolate a double-deletion textitbro-a and bro-c mutant. This shows that the bro-d gene plays an irreplaceable role in the infection of silkworms by this virus ${ }^{21}$.

Blood grasserie, a common disease in Yunnan sericulture regions, presents a serious threat to cocoon production in that province. The Yunnan province located in southwest China with an average elevation of about $2000 \mathrm{~m}$ is abundant in biological, geographical, and ecological diversity. A diverse population of insects could be infected by BmNPV in mulberry orchards. There are various geographical conditions and climate in Yunnan sericulture region, such as Puer, tropical region; HongheMengzi, subtropical region; Qujing, northern subtropical or temperate region; and Dali-Heqing, highaltitude and cold region. Some questions regarding whether these factors may have led to the genetic diversity of BmNPV strains, or that isolate would be capable of causing a major epidemic existence. Here, the collections of 240 samples were based on suspected symptoms of BmNPV infection. Polyhedrin gene, a conservative gene, was used for molecular identification. The bro- $d$ gene was cloned and sequenced. Sequence alignment, phylogenetic analysis based on the ORF of bro-d, and the distribution of BmNPV isolates from Yunnan sericulture regions were determined. This has provided useful data for assessment of the manner of transmission, development of effective control, understanding of the evolution of BmNPV, and the breeding of resistant silkworms suited to Yunnan's various climates.

\section{MATERIALS AND METHODS}

\section{Sample collection and treatment}

According to the geographical distribution of Yunnan sericulture regions, we chose silkworm-raising intensive regions located at different latitudes as collection points. Two-hundred and forty suspected BmNPV disease samples of silkworm were collected and ground with sterilized water. The mixed slurries were then filtered through gauze, and the filtrations were separated by two cycles of differential centrifugation at $4000 \mathrm{~g}$ for $10 \mathrm{~min}$. White polyhedra layers at the bottom of the centrifuge tube were collected, and the polyhedra layers were resuspended in $1 \%$ $(\mathrm{W} / \mathrm{V})$ sodium dodecyl sulphate (SDS) and incubated for $30 \mathrm{~min}$. The polyhedra were then purified by different concentration of sucrose solution (mass fraction $30 \%, 40 \%, 50 \%, 60 \%$ ). 


\section{Pathogen detection of silkworm infected by BmNPV}

The purified polyhedra were suspended in $0.32 \mathrm{M}$ NaCl-0.2 $\mathrm{M} \mathrm{Na}_{2} \mathrm{CO}_{3}$ alkaline solution ( $\left.\mathrm{pH} 10.8\right)$ at $37^{\circ} \mathrm{C}$ for $30 \mathrm{~min}$ to dissolve the polyhedrin matrix. The adequate mixtures were incubated with $1 \mathrm{ml}$ genome extraction fluid $(10 \mathrm{mmol} / \mathrm{l}$ Tris- $\mathrm{HCl}$, pH 8.0; $0.1 \mathrm{~mol} / 1$ EDTA, pH 8.0; 0.5\% SDS) and protease $\mathrm{K}(20 \mathrm{mg} / \mathrm{ml})$. The solutions were then incubated at $55^{\circ} \mathrm{C}$ for $2 \mathrm{~h}$, and viral DNA was extracted once with an equal volume of Tris-saturated phenol buffer-twice with phenol-chloroform-isoamyl alcohol (25:24:1), and once with chloroform. The aqueous phase containing viral genomic DNA was precipitated by absolute ethanol, washed twice with $70 \%$ ethanol, air dried, and resuspended in $100 \mu \mathrm{l}$ of TE buffer (10 mM Tris-HCl, 1 mM EDTA, pH 8.0). These solutions were then used for PCR detection. According to the ORF sequence of the polyhedrin gene, which GenBank logged in as a BmNPV-T3 genome (L33180.1), complete ORF was amplified using the following primers: polh-F, 5'-ATGCCGA ATTATTCATACACCC-3': polh-R, 5,-TTAATACGCC GGACCAGTG-3' in $20 \mu \mathrm{l}$ PCR reactions with the different genome as a template ${ }^{23}$. Reaction system: $10 \times$ PCR buffer $\left(\mathrm{Mg}^{2+}\right.$ plus) $5 \mu \mathrm{l}, 2.5 \mathrm{mM}$ dNTPs $4 \mu \mathrm{l}, 250 \mathrm{U} / \mu \mathrm{l}$ r-Taq polymerase (Takara, Japan) $0.25 \mu \mathrm{l}, 10 \mu \mathrm{mol} / \mathrm{l}$ forward and reverse primers $1 \mu \mathrm{l}$, genomic DNA template $1 \mu \mathrm{l}$, add distilled water to $20 \mu \mathrm{l}$ was used. PCR conditions: $94^{\circ} \mathrm{C}$ predenaturation for $3 \mathrm{~min} ; 94^{\circ} \mathrm{C}$ denaturation for $30 \mathrm{~s}$; annealing $55^{\circ} \mathrm{C}$ for $30 \mathrm{~s} ; 72^{\circ} \mathrm{C}$ for 1 min, 30 cycles; final extension $72^{\circ} \mathrm{C}$ for $10 \mathrm{~min}$. The PCR products were detected on $1 \%$ agarose gel.

\section{Cloning and sequencing of bro-d gene}

The bro- $d$ ORF was amplified using the following primers ${ }^{23}$ : bro- $d$-F, $5^{\prime}$-TATTGCGCCGCAGGAAGCC AT- $3^{\prime}$ : bro- $d$-R, $5^{\prime}$-CGAATCGTCACGCGTCGTTGT A-3', $50 \mu \mathrm{l}$ PCR reactions with Ex-Taq polymerase (Takara, Japan). PCR products were purified using a Sangon Gel Extraction Kit (Sangon Biotech, China Shanghai) and ligated into the cloning vector pMD19-T, then sequenced by Sangon Biotech to confirm the ORF sequences of the bro-d genes of each isolate.

\section{Sequence alignment and phylogenetic analysis}

The bro- $d$ ORF sequences of different BmNPV isolates from Yunnan silkworm-raising regions were used for sequence alignment and phylogenetic analysis. Alignments of bro-d sequences were carried

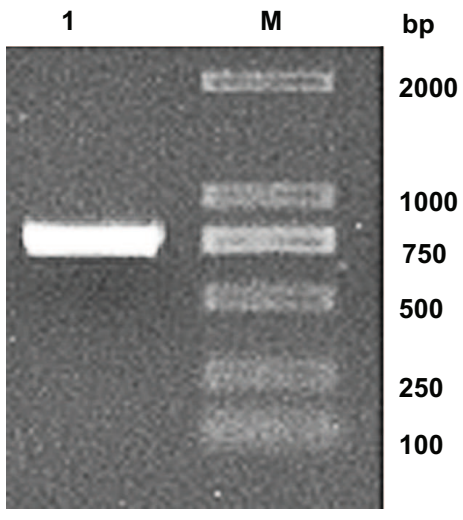

Fig. 1 polh PCR amplification of diseased silkworm genome. 1: diseased silkworm genome; M: DNA marker DL2000 marker.

out using BIOXM 2.6 software. The bro-d gene homology was analysed using DNAMAN 6.0 software. The bro- $d$ nucleotide sequences of BmNPVT3 (L33180.1), BmNPV-Thailand (JN862927.1), B. mandarina nucleopolyhedrovirus S1 (BomaNPVS1, FJ882854.1), BomaNPV-S2 (JQ071499.1), and Lymantria dispar multiple nucleopolyhedrovirus isolate 2161 (LdMNPV-2161, KF695050.2) were downloaded from the NCBI database. BmNPV-T3 and BmNPV-Thailand were selected as representative strains for comparison. To understand the genetic relationship between BomaNPV and BmNPV, and $B$. mori domesticated from B. mandarina, the BomaNPV-S1 and the BomaNPV-S2 were selected as representative strains. Phylogenetic analysis of aligned nucleotide sequences generated from the isolates was carried out using MEGA 5.0 software package (MEGA Software, US) based on the Kimura 2-parameter model under the Neighbour Joining (NJ) method and adjustment of 1000 times the bootstrap ${ }^{24}$. The homologous sequence of LdMNPV2161 was used as outgroup.

\section{RESULTS}

\section{Isolation and identification of BmNPV in different areas}

Two-hundred and forty samples of diseased silkworm were collected from different silkwormraising regions of the Yunnan province, and genomic DNA was extracted from each sample. PCR was performed using specific primers of the polyhedrin gene; the size of PCR products was the same as the polyhedrin gene by electrophoresis detection (approximately 738 bp, shown in Fig. 1). PCR results showed that 233 of the 240 samples were positive. 


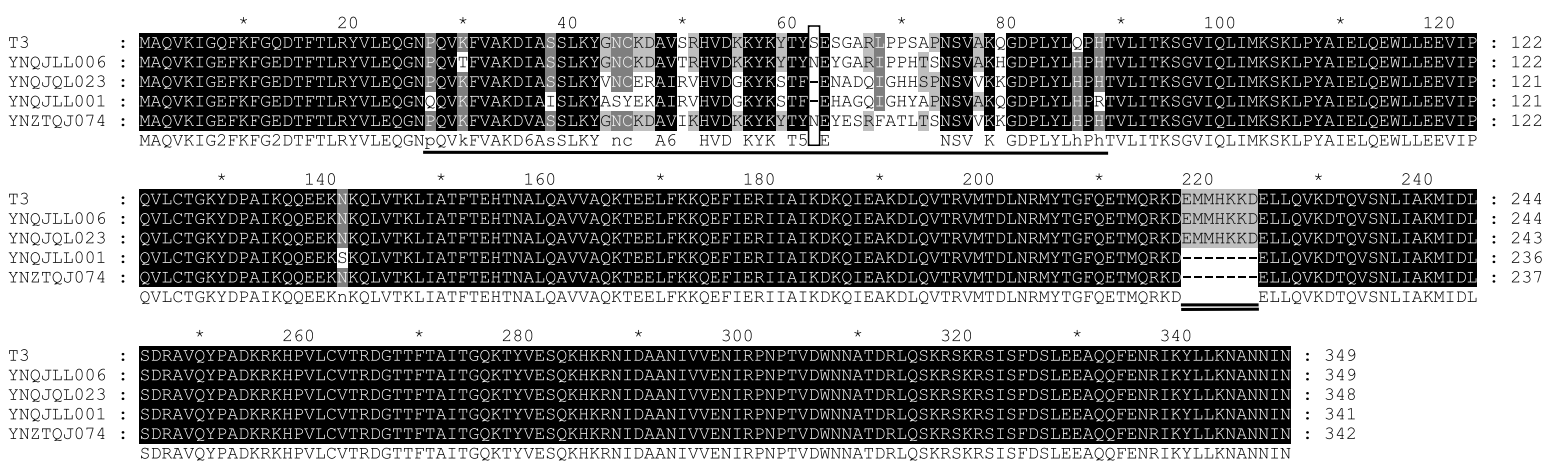

Fig. 2 Alignment of BRO-d amino acid sequence of BmNPV isolates. Identical and similar amino acid residues were indicated by black and grey highlights, respectively. The numbers indicated the position of the amino acid sequence. Underline indicated main different regions of amino acid sequences, rectangle indicated the position of $\mathrm{N}^{62}$ amino acid, double underline indicated the deletions of 7 amino acids located in 217-224 aa region.

Table 1 BmNPV isolates detected by PCR method.

\begin{tabular}{lcc}
\hline Source & $\begin{array}{c}\text { No. of } \\
\text { samples }\end{array}$ & $\begin{array}{c}\text { Positive No. } \\
\text { of BmNPV }\end{array}$ \\
\hline Kunming & 6 & 6 \\
Qujing & 64 & 61 \\
Puer & 41 & 38 \\
Zhaotong & 23 & 23 \\
Dali & 31 & 30 \\
Honghe & 12 & 12 \\
Chuxiong & 51 & 50 \\
Diqing & 1 & 1 \\
Baoshan & 8 & 8 \\
Lijiang & 1 & 1 \\
Wenshan & 1 & 1 \\
Lincang & 1 & 1 \\
\hline Total & 240 & 233 \\
Positive rate & - & $97 \%$ \\
\hline
\end{tabular}

Detection rate of about 97\% (Table 1) furtherly confirmed that the widespread blood grasserie had occurred in Yunnan sericulture regions.

According to geographical distribution of Yunnan sericulture regions and outbreak situation of the blood pathogens of grasserie, 56 genomic samples which had been already identified by PCR were selected to experimental object. Among the 233 positive BmNPV genomes, $56 \mathrm{BmNPV}$ diseased samples were from different counties with the condensed silkworm husbandry in Yunnan Province (Table 2).

\section{Sequence analysis of bro-d gene}

The sequences of bro- $d$ genes were submitted to GenBank, and the accession numbers were presented in Table 2. Comparison of the bro-d coding region sequence showed that the similarity of bro$d$ nucleotide sequence was above $91 \%$ among the different BmNPV isolates (Table 3). BmNPV-T3 bro$\mathrm{d}$ ORF consisted of $1050 \mathrm{nt}$ and encoding 349 amino acid residues. The full length of bro- $d$ ORF of the BmNPV-Thailand strain was $1047 \mathrm{nt}$. The ORF analysis indicated that the full length of bro- $d$ had mostly been $1050 \mathrm{nt}$ and $1047 \mathrm{nt}$ among the 56 different BmNPV isolates, whereas $1026 \mathrm{nt}$ was found in YNQJLL001 and YNPEMJ067. Base insertions of ORF had not been detected. The C-terminal of BRO$\mathrm{d}$ amino acid sequences were highly conserved, whereas the differences existed mainly in other regions ${ }^{21}$. Amino acid sequence alignment of BRO$\mathrm{d}$ among the BmNPV-T3 strain and YNQJLL001, YNQJLL006, YNQJQL023, YNZTQJ074 isolates, the differences in amino acid sequence located in 2788 aa; deletions of 7 amino acids occurred in 217224 aa between YNQJLL001 and YNZTQJ074 were found. $\mathrm{N}$ or Asparagine at the amino acid position 62 was absent in both YNQJLL001 and YNQJQL023 isolates (Fig. 2).

\section{Phylogenetic tree analysis}

Genetic diversity of NPV was mainly caused by bro gene. The bro-d gene was a highly conserved gene in insects NPV genome and necessary for viral replication. Base on the coding regions of the bro- $d$ gene, evolutionary relationship of BmNPV isolates was analysed by NJ method (Fig. 3). The phylogenetic tree revealed that BmNPV strains in the Yunnan silkworm-raising regions had significant genetic diversity. The BmNPV isolates were separated into four main clades: Clade I contained 32 local epidemic strains and was the branch of most members. 
Table 2 Origins of BmNPV isolates from Yunnan used in this study.

\begin{tabular}{|c|c|c|c|}
\hline Isolate & District & $\begin{array}{c}\text { Collection } \\
\text { date }\end{array}$ & $\begin{array}{c}\text { GenBank } \\
\text { No. }\end{array}$ \\
\hline YNQJQL001 & Qujing Qilin & 2012.10 & KX507164 \\
\hline YNQJLL006 & Qujing Luliang & 2012.10 & KX507165 \\
\hline YNQJQL010 & Qujing Qulin & 2013.09 & KX507166 \\
\hline YNQJQL023 & Qujing Qilin & 2013.10 & KX507167 \\
\hline YNQJZY026 & Qujing Zhanyi & 2013.09 & KX507168 \\
\hline YNPEJD037 & Puer Jingdong & 2012.10 & KX507169 \\
\hline YNPEJD051 & Puer Jingdong & 2012.10 & KX507170 \\
\hline YNPEJD055 & Puer Jingdong & 2013.08 & KX507171 \\
\hline YNPENE057 & Puer Ninger & 2013.08 & KX507172 \\
\hline YNPEZY058 & Puer Zhenyuan & 2013.08 & KX507173 \\
\hline YNPEZY059 & Puer Zhenyuan & 2013.08 & KX507174 \\
\hline YNPEZY060 & Puer Zhenyuan & 2013.08 & KX507175 \\
\hline YNPELC062 & Puer Lancang & 2013.08 & KX507176 \\
\hline YNPELC064 & Puer Lancang & 2013.08 & KX507177 \\
\hline YNPEMJ067 & Puer Mojiang & 2013.08 & KX507178 \\
\hline YNPEMJ068 & Puer Mojiang & 2013.08 & KX524427 \\
\hline YNPEMJ071 & Puer Mojiang & 2013.08 & KX524428 \\
\hline YNZTQJ074 & Zhaotong Qiaojia & 2012.10 & KX524429 \\
\hline YNDLHQ091 & Dali Heqing & 2013.09 & KX524430 \\
\hline YNDLHQ093 & Dali Heqing & 2013.09 & KX524431 \\
\hline YNDLHQ096 & Dali Heqing & 2012.10 & KX524432 \\
\hline YNDLHQ097 & Dali Heqing & 2013.09 & KX524433 \\
\hline YNDLXY109 & Dali Xiangyun & 2013.09 & KX524434 \\
\hline YNDLXY116 & Dali Xiangyun & 2013.09 & KX524435 \\
\hline YNHHKY119 & Honghe Kaiyuan & 2013.09 & KX524436 \\
\hline YNCXDG122 & Chuxiong Donggua & 2013.09 & KX524437 \\
\hline YNCXYR139 & Chuxiong Yongren & 2013.10 & KX524438 \\
\hline YNCXYR143 & Chuxiong Yongren & 2013.10 & KX524439 \\
\hline YNCXYR145 & Chuxiong Yongren & 2013.10 & KX524440 \\
\hline YNCXYR148 & Chuxiong Yongren & 2013.10 & KX524441 \\
\hline YNCXYA149 & Chuxiong Yaoan & 2013.10 & KX524442 \\
\hline YNCXYA152 & Chuxiong Yaoan & 2013.10 & KX524443 \\
\hline YNCXYA154 & Chuxiong Yaoan & 2013.10 & KX524444 \\
\hline YNCXYA155 & Chuxiong Yaoan & 2013.10 & KX524445 \\
\hline YNCXYA157 & Chuxiong Yaoan & 2013.10 & KX524446 \\
\hline YNCXYA158 & Chuxiong Yaoan & 2013.10 & KX524447 \\
\hline YNCXDY161 & Chuxiong Dayao & 2013.10 & KX524448 \\
\hline YNCXDY167 & Chuxiong Dayao & 2013.10 & KX524449 \\
\hline YNDQXGLL172 & Diqing Xianggelila & 2013.10 & KX524450 \\
\hline YNBSLY173 & Baoshan Longyang & 013.10 & KX524451 \\
\hline YNBSCN176 & Baoshan Changning & 2013.10 & KX524452 \\
\hline YNBSCN180 & Baoshan Changning & 2013.10 & KX524453 \\
\hline YNKMXD182 & Kunming Xundian & 2012.10 & KX524454 \\
\hline YNKMYL183 & Kunming Yiliang & 2013.07 & KX524455 \\
\hline YNHHYY185 & Honghe Yuanyang & 2013.07 & KX524456 \\
\hline YNLJYS191 & Lijiang Yongsheng & 2014.07 & KX524457 \\
\hline YNZTLD193 & Zhaotong Ludian & 2014.07 & KX524458 \\
\hline YNHHMZ201 & Honghe Mengzi & 2014.09 & KX524459 \\
\hline YNBS205 & Honghe Mengzi & 2012.01 & KX524460 \\
\hline YNHHYY206 & Honghe Yuanyang & 2014.09 & KX524461 \\
\hline YNCXSB207 & Chuxiong Shuangbai & 2014.09 & KX524462 \\
\hline YNLCGM208 & Lincang Gengma & 2014.09 & KX524463 \\
\hline YNPELC211 & Puer Liancang & 2014.05 & KX524464 \\
\hline YNPEZY212 & Puer Zhenyuan & 2014.05 & KX524465 \\
\hline YNQJXW213 & Qujing Xianwei & 2014.05 & KX524466 \\
\hline YNDLXG241 & Dali Xiaguan & 2014.09 & KX524467 \\
\hline
\end{tabular}

Clade IV was a branch of the least number of members and contained 3 epidemic strains. Clades II and III contained 5 and 16 strains, respectively. BmNPVT3 and BmNPV-Thailand distributed in clade III and clade I were selected as representative isolates for comparison. BomaNPV-S1 and BomaNPV-S2 which parasitized $B$. mandarina located in the same clade I branch had a close genetic relationship with BmNPV. Two viruses crossly transmitted between

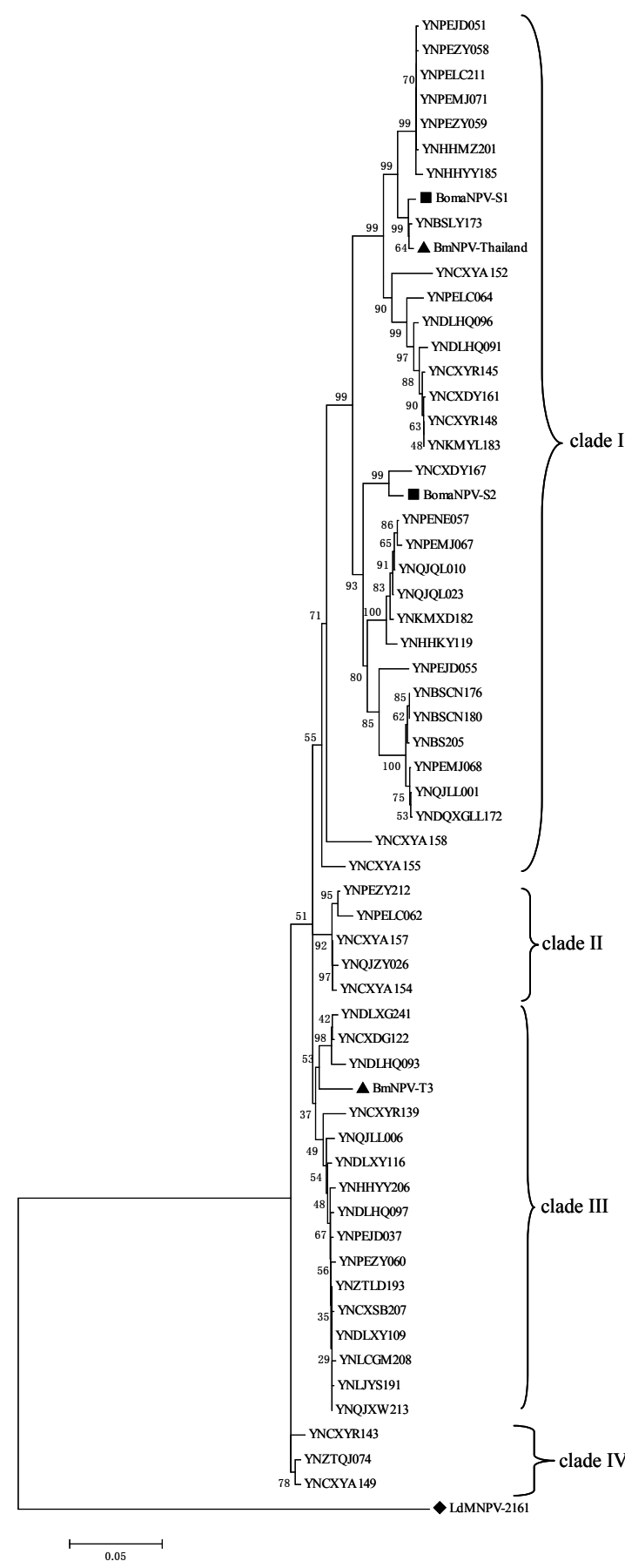

Fig. 3 Phylogenetic tree based on the bro-d gene of BmNPV isolates. Black triangles represented BmNPV bro$d$, as benchmark. Black square represented BomaNPV bro-d. Black rhombus represented LdMNPV bro-d, as outgroup. Numbers above branches indicated reliability from bootstrapping test using 1000 replicates. The bar represented a genetic distance of 0.05 . 


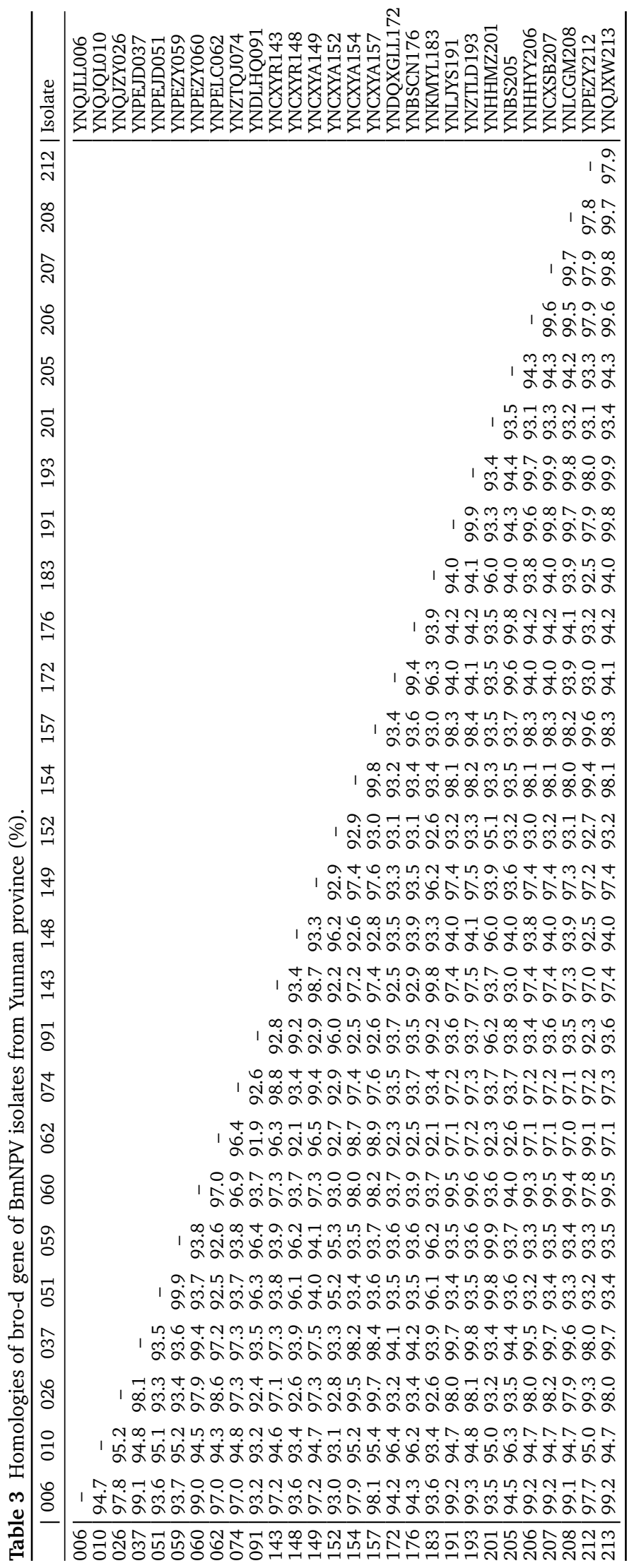




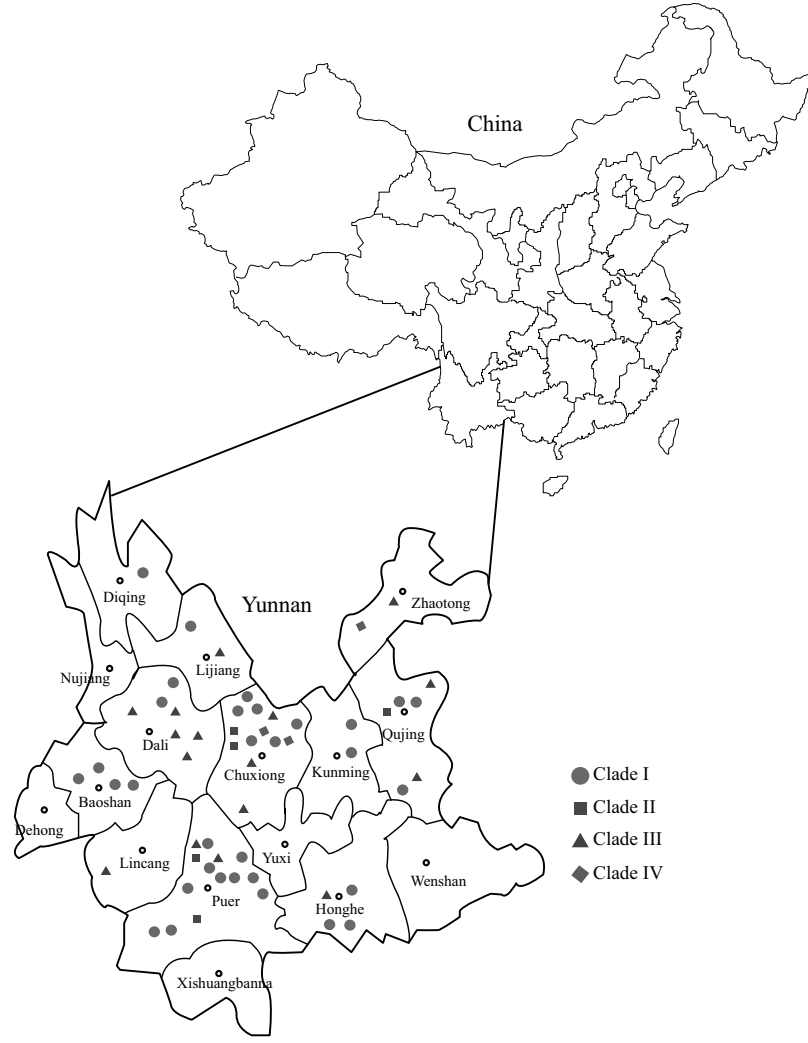

Fig. 4 Geographical distribution of the BmNPV isolates from Yunnan. The distribution of $56 \mathrm{BmNPV}$ isolates in clade I $(\mathbf{\bullet})$, clade II $(\boldsymbol{\square})$, clade III $(\boldsymbol{\Delta})$ and clade IV $(\boldsymbol{\diamond})$.

B. mori and B. mandarina ${ }^{25}$, leading to speculation that either the BomaNPV parasitized B. mandarina from a local mulberry field was an ancestor of the BmNPV source, or two virus types had originated from the same ancestor. BmNPV and BomaNPV were single-grain embedding virus, while LdMNPV was a multiple-grain embedding virus; there were distant relatives between the two kinds of viruses.

\section{Geographical distribution of Yunnan BmNPV isolates}

Geographical distribution of different BmNPV isolates in each inferior branch of clade I was relatively concentrated and adjacent, mainly distributed in Kunming, Qujing, Puer, Baoshan, Dali, Chuxiong, Honghe, and Diqing. Local transmission of this pathogen clearly occurred in the silkworm-raising regions in Yunnan. The isolates of other clades were irregularly scattered throughout Yunnan (Fig. 4), which suggested that long-distance transmission might have occurred. The isolates of clade II were mainly distributed in Puer, Chuxiong, and Qujing.
The regional climate was quite different among the three areas. The genetic relationship of BmNPV isolates within those locations had no direct contact; only clade IV contained YNCXYR143, YNZTQJ074, and YNCXYA149, and three members had distant relatives with other strains.

\section{DISCUSSION}

The silkworm industry is a traditional, pillar industry in China that makes important contributions to the development of its national economy and social culture. Sericulture is also a speciality industry in the Yunnan province. Located in southwest China, it has a variety of ecological environments, such as tropical regions in Puer, subtropical regions in Mengzi, semi-tropics or temperate zone regions in northern Qujing, and high elevation regions in Heqing. Sericulture regions are widely distributed here, as these suitable climatic conditions are conducive to silkworm breeding. This climate is also favourable for the breeding and spread of viral disease. The blood pathogens associated with grasserie outbreaks may cause serious economic losses in areas of Yunnan sericulture. The Yunnan area is particularly affected by the silkworm $B$. mori viral disease during the summer and fall seasons. This study focused on samples collected between 2012 and 2014. The blood pathogens associated with grasserie do the most damage in Puer and Honghe, which are humid, tropical parts of Yunnan, though some temperate zones also showed infestation. The viral disease mainly broke out in fifth-instar silkworm larvae. It was rarely seen in younger worms. The diseased silkworm samples were mainly collected from local households. Silkgrower may overlook diseased silkworms during silkworm husbandry, and the potency of various treatment technologies were not enough to cut off the BmNPV route of transmission in a timely manner. There was an explosion of the disease because the viral particles remained in the environment for a long time.

PCR technology is widely used to rapidly diagnose pathogenic microorganisms, such as in the parasitic silkworm, as it holds the characteristics of rapid trace and sensitive specificity. The design of specific primers is the key to use PCR for diagnosis. The polyhedrin gene was a candidate gene for BmNPV identification ${ }^{26}$. Tang detected infected silkworms through BmNPV polyhedrin gene specific primers successfully ${ }^{23}$. In this study, PCR was used for rapid detection of the collected 240 samples of sick silkworm, 233 were positive with BmNPV 
infection. The detection rate is as high as $97 \%$. If measures are not taken to improve the disinfection of silkworm diseases, the blood pathogens associated with grasserie can sustain an outbreak for a long time in areas of sericulture in Yunnan.

Bro- $d$ is a highly important gene expressed during early stages of viral replication. Bideshi found there to be considerable homology between single copies of the bro gene (ac-bro) in the AcMNPV and BmNPV bro- $d^{14}$. The ac-bro gene was found to directly and indirectly influence the number of viral particles in infected hosts, which suggests that bro$d$ is related to virulence in more than one BmNPV isolate. The variations in the coding region of bro- $d$ gene may cause differences in virulence in different BmNPV isolates. An assessment of homology showed that the nucleotide similarity of bro-d exceeded 91\% in 56 BmNPV isolates (Table 3) and a minimum of the bro- $d$ homology was $92 \%$ between YNPELC062 and YNDLHQ091. The full length of bro-d ORF was 1050 bp from BmNPV-Guangxi (JQ991011.1) and BmNPV-Zhejiang (JQ991008.1) of China local BmNPV epidemic isolates, and the bro-d gene ORF of BmNPV-Thailand was $1047 \mathrm{bp}$. The full lengths of bro-d ORFs were mainly $1050 \mathrm{bp}$ and $1047 \mathrm{bp}$ among the $56 \mathrm{BmNPV}$ isolates, whereas 1026 bp were in YNQJLL001 and YNPEMJ067. The ones of YNZTQJ074, and YNCXYA155 were 1029 bp. The genetic phenomenon of base deletion and insertion had not been found in the pandemic isolates. While compared the bro-d ORF sequence of BmNPV-T3 strain with YNQJLL001 and YNZTQJ074, there was the deletion of 7 amino acids occurred at 217-224 aa. The differences of BRO$\mathrm{d}$ protein mainly focused on the N-terminal. BRO proteins have been identified as nucleocytoplasmic shuttle proteins in CRM-1-mediated nuclear export pathways because the leucine-rich region in their $\mathrm{N}$ terminals plays the same role as CRM-1-dependent nucleus export signal ${ }^{27}$. For the same gene, the absence and insertion of gene fragment could affect the virulence of different virus isolates ${ }^{28}$; for example, the absence of these amino acids of BRO-d among the different BmNPV isolates. However, further work must be performed to determine whether the absence of these amino acids truly affects the virulence of the virus.

Yunnan province has a unique geographic position, the silkworm-raising areas distributed in dam, hills and other uplands. These silkworm-raising regions include both areas with a long history situated in optimal climate and new silkworm-raising regions situated in moist, hot areas. BmNPV iso- lates showed a large amount of genetic diversity because of this broad geographic variety. The genetic differences in BmNPV mainly concerned the bro gene families ${ }^{29}$. The genetic diversity of the virus was mainly caused by the bro gene ${ }^{30}$. Bro$d$ was found to be a highly conserved gene from insect NPV. The $56 \mathrm{BmNPV}$ isolates from Yunnan were separated into four main clades (I, II, III, and IV). This bro-d gene phylogenetic tree contained four clades that had 32, 5, 16, and 3 local epidemic isolates, respectively. The geographical location of $56 \mathrm{BmNPV}$ isolates indicated that the co-relation between evolution of bro- $d$ and geographical location was not obvious. The geographic distribution of clade I isolates in Yunnan showed a concentrated pattern. Local transmission of this pathogen took place in these silkworm-raising regions. The isolates from the other three clades were irregularly scattered throughout Yunnan, which suggested that long-distance transmission was taking place. This phenomenon may have been caused by the dissemination medium, mulberry field insects, human factors and others. Clade IV contained three BmNPV isolates, the samples of YNCXYR143, YNZTQJ074, and YNCXYA149 were collected in Menghu town, Yongren County; Dongchuan town, Yaoan County within the Chuxiong autonomous prefecture, and Baohetan township, Qiaojia County in Zhaotong prefecture-level city, respectively. The topography and climate of these three sericulture regions were very similar. Three pandemic isolates retained the original genetic information, and their more distant relatives contained other BmNPV isolates.

This work presents previously undiscovered molecular epidemiological data concerning BmNPV in China's Yunnan Province. The study mainly includes PCR detection of diseased silkworm samples from sericulture regions and analysis of the genetic relationships among BmNPV strains based on bro-d genes. These results may provide a better evaluation of epidemic regularity of BmNPV and facilitate the discovery of new method of curb the spread of the virus in areas of sericulture within Yunnan. So far, there are no effective drugs for preventing or treating BmNPV infection pathogens accumulated in the natural environment of the Yunnan sericulture area. Hence the process of silkworm feeding, reducing the spread of virus particles and strengthening environmental disinfection are helpful to decrease the incidence of BmNPV disease. In addition, adequately mastering the molecular mechanisms of BmNPV pathogenicity is the key to prevent BmNPV infection for sericulture. 
Acknowledgements: This study was supported by the Foundation for the National Natural Science Foundation of China (Grant No. 31260583 and No. 31560675). We would like to thank members of the research group on pathology of silkworm and all the silkgrowers in the silkworm-raising regions.

\section{REFERENCES}

1. Miele SAB, Garavaglia MJ, Belaich MN, Ghiringhelli PD (2011) Baculovirus: molecular insights on their diversity and conservation. Int J Evol Biol, ID 379424, $1-15$.

2. Jin W (2001) Silkworm pathology. In: Gong CL (ed) Viral Disease, China Agriculture Press, Peking, pp 44-58.

3. Ye MQ, Yang Q, Wu FQ, Li QR, Xiao Y, Kuang ZS, Zhao XJ, Luo GQ (2013) Progress in breeding BmNPV resistant varieties of Bombyx mori. Chinese $J$ Appl Entomol 50, 1447-1452.

4. Bai XR, Ran RF, Dong ZP, Dong JH, Huang P (2010) Study on virulence of BmNPV to Bombyx mori in different areas of Yunnan. Southwest China J Agr Sci 23, 2098-2101.

5. Keddie BA, Aponte GW, Volkman LE (1989) The pathway of infection of Autographa californica nuclear polyhedrosis virus in an insect host. Science 243, 1728-1730.

6. Qi YP, Liu D, Sun XJ (2000) Comparison study about the vp39 gene of Bombyx mori nuclear polyhedrosis virus two isolates from China and Japan. Virol Sin 15, $52-58$.

7. Hong HK, Woo SD, Choi JY, Lee HK, Kim MH, Je YH, Kang SK (2000) Characterization of four isolates of Bombyx mori nucleopolyhedrovirus. Arch Virol 145, 2351-2361.

8. Liang X, Lu ZL, Wei BX, Feng JL, Qu DC, Luo TR (2013) Phylogenetic analysis of Bombyx mori nucleopolyhedrovirus polyhedrin and p10 genes in wild isolates from Guangxi Zhuang Autonomous Region, China. Virus Genes 46, 140-151.

9. Lopez-Bueno A, Mateu MG, Almendral JM (2003) High mutant frequency in populations of a DNA virus allows evasion from antibody therapy in an immunodeficient host. $J$ Virol 77, 2701-2708.

10. Katsuma S, Kawaoka S, Mita K, Shimada T (2008) Genome-wide survey for baculoviral host homologs using the Bombyx genome sequence. Insect Biochem Mol Biol 38, 1080-1086.

11. Long M, Betran E, Thornton K, Wang W (2003) The origin of new genes: glimpses from the young and old. Nat Rev Genet 4, 865-875.

12. Pang M, Pan GQ, Li T, Wang X, Zhou ZY (2007) The analysis of bro genes of GD isolate of Bombyx mori nucleopolyhedrovirus. Chinese J Virol 23, 485-489.

13. Ayres MD, Howard SC, Kuzio J, Lopez-Ferber M, Possee RD (1994) The complete DNA sequence of
Autographa californica nuclear polyhedrosis virus. Virology 202, 586-605.

14. Bideshi DK, Renault S, Stasiak K, Federici BA, Bigot Y (2003) Phylogenetic analysis and possible function of bro-like genes, a multigene family widespread among large double-stranded DNA viruses of invertebrates and bacteria. J Gen Virol 84, 2531-2544.

15. Harrison RL, Bonning BC (2003) Comparative analysis of the genomes of Rachiplusia ou and Autographa californica multiple nucleopolyhedroviruses. J Gen Virol 84, 1827-1842.

16. Kuzio J, Pearson MN, Harwood SH, Funk CJ, Evans JT, Slavicek JM, Rohrmann GF (1999) Sequence and analysis of the genome of a baculovirus pathogenic for Lymantria dispar. Virology 253, 17-34.

17. Gomi S, Majima K, Maeda, S (1999) Sequence analysis of the genome of Bombyx mori nucleopolyhedrovirus. J Gen Virol 80, 1323-1337.

18. Jakob NJ, Müller K, Bahr U, Darai G (2001) Analysis of the first complete DNA sequence of an invertebrate iridovirus: coding strategy of the genome of Chilo iridescent virus. Virology 286, 182-196.

19. Zhou JB, Li XQ, De-Eknamkul W, Suraporn S, Xu JP (2012) Identification of a new Bombyx mori nucleopolyhedrovirus and analysis of its bro gene family. Virus Genes 44, 539-547.

20. Xu YP, Cheng RL, Xi Y, Zhang CX (2013) Genomic diversity of Bombyx mori nucleopolyhedrovirus strains. Genomics 102, 63-71.

21. Kang W, Suzuki M, Zemskov E, Okano K, Maeda S (1999) Characterization of Baculovirus Repeated Open Reading Frames (bro) in Bombyx mori Nucleopolyhedrovirus. $J$ Virol 73, 10339-10345.

22. Chen F, Wang JM, Sun BG, Chen XM, Yang ZX, Duan ZY (2012) Relationship between geographical distribution of Pinus yunnanensis and climate. Fore Res 25, 163-168.

23. Tang FF, Zhang YH, Shao YL, Zhu F, Huang P, Bai XR (2017) Isolation and identification of a new Bombyx mori nucleopolyhedrovirus strain isolated from Yunnan, China. Scienceasia 43, 26-32.

24. Tamura K, Peterson D, Peterson N, Stecher G, Nei M, Kumar S (2011) MEGA5: molecular evolutionary genetics analysis using maximum likelihood, evolutionary distance, and maximum parsimony methods. Mol Biol Evol 28, 2731-2739.

25. Xu YP, Ye ZP, Niu CY, Bao YY, Wang WB, Shen WD, Zhang CX (2010) Comparative analysis of the genomes of Bombyx mandarina and Bombyx mori nucleopolyhedroviruses. J Microbiol 48, 102-110.

26. Lange $M$, Wang $H$, Zhihong $H$, Jehle JA (2004) Towards a molecular identification and classification system of lepidopteran-specific baculoviruses. Virology $325,36-47$.

27. Kang W, Kurihara M, Matsumoto S (2006) The BRO proteins of Bombyx mori nucleopolyhedrovirus are nucleocytoplasmic shuttling proteins that utilize the 
CRM1-mediated nuclear export pathway. Virology 350, 184-191.

28. Kane LP, Mollenauer MN, Weiss A (2004) A prolinerich motif in the $\mathrm{C}$ terminus of Akt contributes to its localization in the immunological synapse. $J \mathrm{Im}$ munol 172, 5441-5449.

29. Ferber ML, Argaud O, Croizier L, Croizier G (2001) Diversity, distribution, and mobility of bro gene se- quences in Bombyx mori nucleopolyhedrovirus. Virus Genes 22, 247-254.

30. Harrison RL, Popham HJ, Breitenbach JE, Rowley DL (2012) Genetic variation and virulence of Autographa californica multiple nucleopolyhedrovirus and Trichoplusia ni single nucleopolyhedrovirus isolates. $J$ Invertebr Pathol 110, 33-47. 